\title{
COVID-19-Related National Re-confinement: Recommendations From the National French Observatory for Physical Activity and Sedentary Behaviors (ONAPS)
}

\author{
David Thivel, Michéle Tardieu, Pauline Genin, Alicia Fillon, Benjamin Larras, Pierre Melsens, \\ Julien Bois, Frédéric Dutheil, Francois Carré, Gregory Ninot, Jean-Francois Toussaint, \\ Daniel Rivière, Yves Boirie, Bruno Pereira, Angelo Tremblay, and Martine Duclos
}

While a declined physical activity level (PAL) and an increase of sedentary behaviors at all ages during the COVID-19-induced first confinement period have been unanimously observed worldwide, the lately instituted new social distancing strategies and lockdowns around the world require the formulation and dissemination of adapted and accessible recommendations to promote physical activity (PA) and limit the progression of sedentary time (SED) in the upcoming weeks. From a public health perspective, it appears imperative to encourage the adoption of healthy movement behaviors despite the new activity restrictions imposed by governments to slow down the alarming progression of the SARS-CoV-2 pandemic.

On top of their well-known impact on physical and metabolic health in both healthy individuals and patients, ${ }^{1}$ both PA and sedentary behaviors have been shown to be strongly associated (in an opposite way) with depression, anxiety, stress, and overall wellbeing. ${ }^{2-4}$ Decline of PA and increase of SED during the first confinement period have been clearly related to a deterioration of several mental health indicators at all ages. ${ }^{5-9}$ Moreover,

Thivel, Tardieu, Genin, Fillon, Larras, Melsens, Bois, Carré, Ninot, Toussaint, Rivière, Boirie, Pereira, Tremblay, and Duclos are with the National Observatory for Physical Activity and Sedentary Behaviors (ONAPS), Clermont-Ferrand, France. Dutheil is with Occupational Medicine, University Hospital CHU G. Montpied, Clermont-Ferrand, France; University Clermont 1, UFR Medicine, ClermontFerrand, France; and Université Clermont Auvergne, CNRS, LaPSCo, Physiological and Psychosocial Stress. Thivel is also with Clermont Auvergne University, EA 3533, Laboratory of the Metabolic Adaptations to Exercise under Physiological and Pathological Conditions (AME2P), CRNH Auvergne, Clermont-Ferrand, France. Bois is also with the Universite de Pau \& des Pays de l'Adour, e2s UPPA, MEPS, Tarbes, France. Boire and Duclos are also with University Clermont 1, UFR Medicine, Clermont-Ferrand, France. Carré is also with Cardiologie et maladies vasculaires, médecine du sport, CHU Pontchaillou, université Rennes 1, Inserm 1099, Rennes, France. Ninot is also with Unité de recherche IDESP, Université de Montpellier, Institut du Cancer de Montpellier, Montpellier, France. Toussaint is also with the Institut de Recherche Biomédicale et d'Épidémiologie du Sport (IRMES), Institut National du Sport, de l'Expertise et de la Performance (INSEP); EA7329 Institut de Recherche BioMédicale et d'Épidémiologie du Sport (IRMES); and the Centre d'Investigation en Médecine du Sport, Paris, France. Boirie is also with the Departement of Human Nutrition, Clermont-Ferrand University Hospital, G. Montpied Hospital, Clermont-Ferrand, France; and INRA, UMR 1019, Clermont-Ferrand, France. Pereira and Duclos are also with Clermont-Ferrand University Hospital, Biostatistics unit (DRCI), Clermont-Ferrand, France. Duclos is also with the Department of Sport Medicine and Functional Explorations, ClermontFerrand University Hospital, G. Montpied Hospital, Clermont-Ferrand, France. Tremblay is also with the Département de l'éducation Physique, Faculté des Sciences de l'éducation, Université Laval, Québec City, QC, G1V 0A6, Canada. Thivel (david.thivel@uca.fr) is corresponding author. sedentariness plays a major role in COVID-19 mortality worldwide, while inactivity partly determines the proportion of patients requiring intensive care due its subsequent metabolic disorders (obesity, hypertension, diabetes).$^{10}$ It appears then necessary to elaborate effective strategies to maintain the PAL of already active individuals but also to encourage inactive people to improve their activity, while, obviously, fighting against the instauration of a sort of "supra-sedentariness" favored by social distancing and increased teleworking. Maintaining and encouraging PA during a confinement period appears indeed essential to preserve our health capital but also to reinforce our immunity, which seems actually of high importance to face the COVID-19 virus.

In order to face this worrying situation induced by the first quarantine period related to the COVID-19 virus, the World Health Organization (WHO) proposed some recommendations aimed at improving individuals' total daily activity level. ${ }^{11,12}$ Briefly, WHO encouraged adults to increase their household chores, to integrate online PA programs, to exercise while listening to enjoyable music, to climb stairs as much as possible and to perform resistance exercises. For children and adolescents (5 to 17 years old), WHO recommended participation in online physical activities, active plays, indoor challenging games, the realization of activities mobilizing muscle groups, and the development of new motor abilities and skills.

Since the first confinement, some authors proposed adapted PA and SED recommendations for older adults, ${ }^{13-15}$ for children and adolescents, ${ }^{14-16}$ and for the overall population. ${ }^{17-19}$ Briefly, all these recommendations underlined the need for home-based adapted versions of the usual PA recommendations, proposing adequate durations and intensities and stressing the particular need to adopt some sedentary break strategies.

The French National Observatory for Physical Activity and Sedentary Behaviors (ONAPS), altogether with the French Minister of Sports, conducted one of the largest worldwide surveys evaluating the impact of the first confinement on PA and SED. ${ }^{20}$ Importantly, this survey questioned the impacts of the lockdown from childhood to elderly and the conducted analysis also explored whether these changes were associated with the initial levels of PA and SED of individuals (for complete and detailed results, see ONAPS' position stand ${ }^{20}$ ). Briefly this survey showed, altogether with an alarming general reduction of PA, a dramatic increase in sitting time per day in nearly $50 \%$ of participants (reaching $+72 \%$ in initially sedentary children and adolescents) and, except in older people, a huge increase in screen time per day (children and adolescents: +62 to $69 \%$, adults: $+57 \%$ ). According to our results, a very large proportion of initially active individuals decreased 
their PAL during the confinement while previously inactive ones tended to start exercising, mainly following online training classes that remain to be considered with caution as non-individualized and adapted to everyone (especially inactive people). Interestingly, people who maintained or increased their PAL during the first days of the previous lockdown were more likely to remain active and less sedentary during the whole confinement (which was positively associated with overall mental health and wellbeing). Our results also pointed out the necessity to help children and adolescents to remain active as they showed a dramatic increase of their overall unhealthy movement behaviors (decreased PAL and increased screen time). The main results of this survey have been previously detailed. ${ }^{20}$

At the dawn of a new generalized episode of confinement, ONAPS proposes here some new key recommendations and advices, based on the actually available evidences and on the results of its COVID-19 national survey, ${ }^{20}$ to prevent the decline in active behaviors and rise in SED that have been observed during the first confinement (from March to May 2020). Importantly, these recommendations and advices (Table 1) are not substitutes to the general PA guidelines, but propose an adapted approach to avoid the already observed negative impacts of a lockdown on our daily active and sedentary behaviors.

While not exhaustive, these advices should be considered as simple strategies proposed to avoid the observed confinementinduced decline of PAL and rise of SED. There is no one-sizefits-all solution, but everyone has to realize the importance of maintaining an active lifestyle despite such particular circumstances. Importantly, according to what was observed during the previous lockdown, initially active people with a low or moderate time devoted to sedentary behaviors are particularly at risk of adopting unhealthy movement behaviors, as well as adolescents, older people, and women.

Although highly restrictive, this new wave of the COVID-19 pandemic and the instituted new confinement (for at least several weeks) should be considered as a potential opportunity to analyze our usual daily activities and sedentary behaviors, and to adopt new habits, on an individual and/or family basis, to improve our PAL, reduce our time spent sedentary, and then enhance our overall mental and physical health.

\section{Table 1 Recommendations and Advices From the French National Observatory for Physical Activity and Sedentary Behaviors (ONAPS)}

Recommendation or Advice

- Favor any daily movement, whatever its intensity and duration, keeping in mind that our overall level of physical activity needs every single and simple movement.

- We encourage the adoption of an active daily lifestyle from the very early days of this new confinement. Similarly, a particular attention should be given to our sedentary behaviors from the first days, to avoid their instauration and adopt healthy movement behaviors and habits.

- As for the first confinement, it remains possible to perform 1 hour of outside physical activity per day. We encourage the adoption of daily walks or runs. Several days a week, we encourage to share these outdoor activities with the other members who compose the family home.

- Initially active individuals must be careful to maintain their level of practice, keeping in mind that our usual daily activities related to active transportation and occupational activities will be drastically decreased and should be compensated for by favoring other kinds of activities such as active plays with kids, walking, and housekeeping.

- In case of telecommuting, it is important to warm up and stretch 5 to 10 minutes before setting up at your desk, emphasizing on the back, shoulders, and pelvis. A warm-up of the main muscle groups is also recommended.

- Initially inactive individuals, or those with a low level of physical activity, should engage in a gradual and a step-by-step approach to progressively reach the recommendations. A brutal engagement in an important volume of physical activity has been shown counterproductive and does not favor a long-term adoption of physical activity behaviors.

- Online physical activity classes can be followed and have to be encouraged keeping in mind that they might not be individualized and that everyone has to auto-adapt their practice based on their own physical and exertion sensations. Exercising does not have to be painful and we have to listen to our own feelings and capacities to progressively improve. A scale of perceived difficulty (0 to 10$)$ can be used instead of proper intensities; a rating between 5 and 7 being appropriate to ensure long-term engagement.

- Parents and legal representatives should encourage physical activity among children and adolescents. Performing active games 1 hour or so per day with our young kids, even combined with 1 hour of outdoor walk or play, is not enough to maintain their daily physical activity level. Regular short blocks of games and activities should be encouraged (which could join up with the active breaks recommended to break our sedentary time).

- In order to break this sedentary time in both youth and parents, we suggest the adoption of active breaks that involve the whole family (or several members), based on small active plays and games. We also encourage the instauration of relatively short periods of relaxing activities such as stretching before and after a whole day of teleworking.

- On top of the recommended active breaks, homemade active desks can be created to turn our usual tables or desk into standing desk, for instance. When possible cycling drives can be purchased. Simple strategies could be adopted such as systematically walking while on the phone.

- Families and peers should regularly, on a daily basis, encourage older people to engage in simple activities such as walking. The government gives the opportunity to visit individuals who necessitate help and support and we believe that the maintenance of our elders' physical activity is a challenge that has to be considered during this new lockdown.

- Importantly, the practice of physical activity should be conditioned to our health status. Fever, persistent fatigue, and other symptoms (related or not to COVID-19), as well as muscle or joint pain, should lead everyone to adapt their activity.

- Patients with chronic disease should not stop physical activity that is an integral part of their treatment. They have to adapt indoor and outdoor physical activity to reach at least 30 minutes of physical activity every day and to break regularly (hourly) sedentary behaviors. 


\section{References}

1. Zhao M, Veeranki SP, Magnussen CG, Xi B. Recommended physical activity and all cause and cause specific mortality in US adults: prospective cohort study. BMJ. 2020;370(1):m203.

2. Netz Y. Is the comparison between exercise and pharmacologic treatment of depression in the clinical practice guideline of the American College of Physicians evidence-based? Front Pharmacol. 2017;8:257. PubMed ID: 28555108 doi:10.3389/fphar.2017.00257

3. Omorou AY, Vuillemin A, Menai M, et al. 10-year cumulative and bidirectional associations of domain-specific physical activity and sedentary behaviour with health-related quality of life in French adults: Results from the SU.VI.MAX studies. Prev Med. 2016;88: 66-72. PubMed ID: 27058941 doi:10.1016/j.ypmed.2016.03.023

4. Stubbs B, Vancampfort D, Thompson T, et al. Pain and severe sleep disturbance in the general population: Primary data and meta-analysis from 240,820 people across 45 low- and middle-income countries. Gen Hosp Psychiatry. 2018;53:52-58. PubMed ID: 29807277 doi: 10.1016/j.genhosppsych.2018.05.006

5. Ugbolue UC, Duclos M, Urzeala C, et al. An assessment of the novel COVISTRESS Questionnaire: COVID-19 impact on physical activity, sedentary action and psychological emotion. J Clin Med. 2020; 9(10):E3352. PubMed ID: 33086648 doi:10.3390/jcm9103352

6. Carriedo A, Cecchini JA, Fernández-Río J, Méndez-Giménez A. Resilience and physical activity in people under home isolation due to COVID-19: a preliminary evaluation. Ment Health Phys Act. 2020; 19:100361. PubMed ID: 33024452 doi:10.1016/j.mhpa.2020.100361

7. Chouchou F, Augustini M, Caderby T, Caron N, Turpin NA, Dalleau G. The importance of sleep and physical activity on well-being during COVID-19 lockdown: reunion island as a case study. Sleep Med. 2021;77:297-301. PubMed ID: 33020037 doi:10.1016/j.sleep.2020. 09.014

8. Werneck AO, Silva DR, Malta DC, et al. Changes in the clustering of unhealthy movement behaviors during the COVID-19 quarantine and the association with mental health indicators among Brazilian adults. Transl Behav Med. 2020;6:ibaa095. doi:10.1093/tbm/ibaa095

9. Alves JM, Yunker AG, DeFendis A, Xiang AH, Page KA. Associations between affect, physical activity, and anxiety among US children during COVID-19. medRxiv. 2020;23:20216424.

10. De Larochelambert Q, Marc A, Antero J, Le Bourg E, Toussaint JF. Covid-19 mortality worldwide: a matter of vulnerability among nations facing limited margins of adaptation. Front Public Health. 2020; 8:604339. PubMed ID: 33330343 doi:10.3389/fpubh.2020.604339
11. World Health Organization. Global Strategy on Diet, Physical Activity and Health. 2020. https://www.who.int/dietphysicalactivity/background/ en/2020. Accessed September 12, 2020.

12. World Health Organization. \#HealthyAtHome - Physical Activity. 2020. https://www.who.int/news-room/campaigns/connecting-theworld-to-combat-coronavirus/healthyathome/healthyathome---physicalactivity. Accessed August 25, 2020.

13. Jiménez-Pavón D, Carbonell-Baeza A, Lavie CJ. Physical exercise as therapy to fight against the mental and physical consequences of COVID-19 quarantine: Special focus in older people. Prog Cardiovasc Dis. 2020;63(3):386-388. PubMed ID: 32220590 doi:10.1016/ j.pcad.2020.03.009

14. Lakicevic N, Moro T, Paoli A, et al. Stay fit, don't quit: geriatric exercise prescription in COVID-19 pandemic. Aging Clin Exp Res. 2020;32(7):1209-1210. PubMed ID: 32449107 doi:10.1007/s40520020-01588-y

15. Hammami A, Harrabi B, Mohr M, Krustrup P. Physical activity and coronavirus disease 2019 (COVID-19): Specific recommendations for home-based physical training [published online ahead of print April 20, 2020]. Manag Sport Leis. doi:10.1080/23750472.2020. 1757494

16. Guan H, Okely AD, Aguilar-Farias N, et al. Promoting healthy movement behaviours among children during the COVID-19 pandemic. Lancet Child Adolesc Health. 2020;4(6):416-418. PubMed ID: 32458805 doi:10.1016/S2352-4642(20)30131-0

17. Ricci F, Izzicupo P, Moscucci F, et al. Recommendations for physical inactivity and sedentary behavior during the coronavirus disease (COVID-19) pandemic. Front Public Health. 2020;8:199. PubMed ID: 32574294 doi:10.3389/fpubh.2020.00199

18. Chtourou H, Trabelsi K, H'mida C, et al. Staying physically active during the quarantine and self-isolation period for controlling and mitigating the COVID-19 pandemic: A systematic overview of the literature. Front Psychol. 2020;11:1708. PubMed ID: 33013497 doi: 10.3389/fpsyg.2020.01708

19. Jurak G, Morrison SA, Leskošek B, et al. Physical activity recommendations during the coronavirus disease-2019 virus outbreak. J Sport Health Sci. 2020;9(4):325-327. PubMed ID: 32426171 doi: 10.1016/j.jshs.2020.05.003

20. Genin PM, Dutheil F, Larras B, et al. Promoting physical activity and reducing sedentary time among tertiary workers: Position stand from the French National Observatory for Physical Activity and Sedentary Behaviors (ONAPS). J Phys Act Health. 2020;16(9):677-678. doi: 10.1123/jpah.2019-0154 